3. - The Riemann-Roch theorem for analytic embeddings, Topology 1 (1962), 151-166.

4. A. Borel and J.-P. Serre, Le théorème de Riemann-Roch (d'après Grothendieck), Bull. Soc. Math. France 86 (1958), 97-136.

5. E. Dyer, Relations between cohomology theories, Aarhus Colloquium on Algebraic Topology, 1962; pp. 89-93.

6. B. Eckmann, Cohomology of groups and transfer, Ann. of Math. (2) 58 (1953), 481-493. 35.

7. - On complexes with operators, Proc. Nat. Acad. Sci. U.S.A. 39 (1953),

8. M. Hall, Jr., Theory of groups, Macmillan, New York, 1959.

The University, Manchester, England and

University of WitwatersRand, Johannesburg, South Africa

\title{
ISOMORPHIC COMPLEXES
}

\section{BY JACK SEGAL}

\section{Communicated by M. L. Curtis, December 28, 1964}

In this paper we show that if $K$ and $L$ are $n$-complexes, then $K$ and $L$ are isomorphic iff the 1-sections of the first derived complexes of $K$ and $L$ are isomorphic. This provides a low-dimensional method for establishing the isomorphism (homeomorphism) of complexes (polyhedra).

Throughout, $s_{p}$ will denote a (rectilinear) $p$-simplex with vertices $a^{0}, a^{1}, \cdots, a^{p} ; K$ will denote a (finite geometric) complex with $n$ section $K^{n}$ and first derived complex $K^{\prime}$. The closed star of a vertex $a$ of $K$, st $(a)$, is the set of simplexes of $K$ having $a$ as a face and all their faces. For more details see [2].

Definition 1. An $n$-complex $K$ is full provided, for any subcomplex $L$ of $K$ which is isomorphic to $s_{p}^{1}, 2 \leqq p \leqq n, L^{0}$ spans a $p$-simplex of $K$.

Theorem 1. Suppose $K$ and $L$ are full $n$-complexes. Then $K$ and $L$ are isomorphic iff $K^{1}$ and $L^{1}$ are isomorphic.

Proof. We need only consider the case when $K^{1}$ and $L^{1}$ are isomorphic. Let $v: K^{1} \rightarrow L^{1}$ be an admissible vertex transformation of $K^{1}$ onto $L^{1}$ with an admissible inverse. Then $a^{0}, a^{1}$ span a 1 -simplex of $K$ iff $v\left(a^{0}\right), v\left(a^{1}\right)$ span a 1-simplex of $L$. Furthermore, for any $p$, $2 \leqq p \leqq n$, if $a^{0}, a^{1}, \cdots, a^{p}$ span a $p$-simplex $s_{p}$ of $K$, then $v\left[s_{p}^{1}\right]$ is isomorphic to $s_{p}^{1}$. So, using the fullness of $L$, we get that $\left(v\left[s_{p}^{1}\right]\right)^{0}$ 
$=\left\{v\left(a^{0}\right), v\left(a^{1}\right), \cdots, v\left(a^{p}\right)\right\}$ spans a $p$-simplex of $L$. Similarly, if $\left\{v\left(a^{0}\right), v\left(a^{1}\right), \cdots, v\left(a^{p}\right)\right\}$ spans a $p$-simplex of $L$, then $\left\{a^{0}, a^{1}, \cdots, a^{p}\right\}$ spans a $p$-simplex of $K$. Hence, $v$ is an admissible vertex transformation of $K$ onto $L$ with an admissible inverse and so $K$ and $L$ are isomorphic.

Lemma 1. If $K$ is an $n$-complex, then $K^{\prime}$ is a full $n$-complex.

Proof. Suppose $L$ is a subcomplex of $K^{\prime}$ and $L$ is isomorphic to $s_{p}^{1}, 2 \leqq p \leqq n$. Then there is a barycenter $b$ of a $q$-simplex of $K, p \leqq q \leqq n$, such that $L \subset \operatorname{st}(b)$. Hence $L^{0}$ spans a $p$-simplex of $K$.

TheOREM 2. If $K$ and $L$ are n-complexes, then $K$ and $L$ are isomorphic iff $\left(K^{\prime}\right)^{1}$ and $\left(L^{\prime}\right)^{1}$ are isomorphic.

Proof. Suppose $K$ and $L$ are isomorphic. Then $K^{\prime}$ and $L^{\prime}$ are isomorphic $n$-complexes. Since they are both full (Lemma 1) we can apply Theorem 1 to get that $\left(K^{\prime}\right)^{1}$ and $\left(L^{\prime}\right)^{1}$ are isomorphic.

Now assume that $\left(K^{\prime}\right)^{1}$ and $\left(L^{\prime}\right)^{1}$ are isomorphic. Then Theorem 1 implies $K^{\prime}$ and $L^{\prime}$ are isomorphic and so $K$ and $L$ are isomorphic (see [1]).

\section{REFERENCES}

1. R. L. Finney, The insufficiency of barycentric subdivision, Notices Amer. Math. Soc. 10 (1963), 668; Michigan Math J. (to appear).

2. P. J. Hilton and S. Wiley, Homology theory, Cambridge Univ. Press, Cambridge, 1960.

UNIVERSITY OF WASHINGTON 